\title{
Utricle-Associated Mucosal Gland Hyperplasia
}

National Cancer Institute

\section{Source}

National Cancer Institute. Utricle-Associated Mucosal Gland Hyperplasia. NCI Thesaurus.

Code C63929.

A benign microacinar proliferation occurring exclusively in the area of the utricle. 